\title{
Circulating miR-21 as a prognostic and predictive biomarker in oral squamous cell carcinoma
}

\author{
Nosheen Mahmood ${ }^{1}$, Muhammad Hanif ${ }^{2}$, Akhtar Ahmed ${ }^{3}$, \\ Qamar Jamal ${ }^{4}$, Shamim Mushtaq ${ }^{5}$, Adnan Khan ${ }^{6}$, Muhammad Saqib ${ }^{7}$
}

\begin{abstract}
Background \& Objective: The high-throughput analysis of circulating microRNAs (miRNAs) is an active area of biomarker research. The oral cancer remains a common cancer among Pakistani males that continues to present at an advance stage, thus exhibiting poor survival. MiRNA 21 (miR-21) is the most consistently over-expressed miRNA in different types of tumor tissues. However, information regarding expression of miR-21 in plasma remains to be resolved. Therefore, present study was designed to investigate if miR-21 was expressed in plasma of patients with oral cancer, and further explore its diagnostic and prognostic potential.

Methods: Present study was conducted at Ziauddin University and Karachi Institute of Radiotherapy and Nuclear Medicine (KIRAN). Histologically confirmed cases of oral squamous cell carcinoma were recruited from Oncology Department of Ziauddin Hospital between 2013 and 2017. Controls were carefully selected after considering age, gender and socioeconomic condition. MiRNA was extracted and immediately reverse transcribed to CDNA. MiR-21 expression was evaluated using probes specifically designed for Real time quantitative polymerase chain reaction.

Results: A significant over expression of miRNA 21 was observed in histologically confirmed cases as compared to controls. The increased expression of miRNA 21 was also reported to be associated with tumor size, metastasis and local invasion $(\mathrm{p}<0.05)$.

Conclusion: The expression of circulating miR-21 in plasma samples of oral cancer patients makes it a promising diagnostic and prognostic marker.
\end{abstract}

KEYWORDS: miRNA, Oral cancer, Real time PCR, miRNA-21.

doi: https://doi.org/10.12669/pjms.35.5.331

How to cite this:

Mahmood N, Hanif M, Ahmed A, Jamal Q, Mushtaq S, Khan A, et al. Circulating miR-21 as a prognostic and predictive biomarker in oral squamous cell carcinoma. Pak J Med Sci. 2019;35(5):1408-1412. doi: https://doi.org/10.12669/pjms.35.5.331

This is an Open Access article distributed under the terms of the Creative Commons Attribution License (http://creativecommons.org/licenses/by/3.0), which permits unrestricted use, distribution, and reproduction in any medium, provided the original work is properly cited.

\section{INTRODUCTION}

Oral cancer contributes to a significant proportion of cancer related morbidity and mortality in Pakistan. ${ }^{1}$ Treatment guidelines are being modified and updated at an escalating rate but survival outcome in advanced stage has failed

\footnotetext{
Correspondence:

Dr. Muhammad Hanif,

Karachi Institute of Radiotherapy and Nuclear Medicine (KIRAN),

Safoora Chawk, University Road,

Karachi, Pakistan.

Email: hanifmuhammad@hotmail.com

* Received for Publication

January 8, 2019

* Revision Received:

* Revision Accepted:

July 1,2019

July 6, 2019
}

to improve over several decades. Most tragic part is that majority of the patients are diagnosed at an advance stage. ${ }^{2}$ In this scenario the major focus of the research should be to identify markers which could predict prognosis and help in streamlining individualized treatment.

MicroRNAs can serve this purpose as recent researches have revealed their potential capability of being used as diagnostic, prognostic and predictive tool in cancers. Promising results has been the main driver of extensive research in the field of microRNAs. Therapeutic interventions that can be made to replace or suppress deregulated miRNAs have added further to the quest of exploring these molecular targets. ${ }^{3}$ Interesting enough miRNAs have 
not only been discovered in tumor tissue but can also be recovered from body fluids including serum and plasma of cancer patients and thus may serve as an important minimally invasive diagnostic tool. ${ }^{4}$

Circulating miRNAs are highly stable, owing to protection against RNAses offered by modifications like uridinylation, adenylation and methylations. Moreover, enclosure in micro vesicles and binding to RNA binding proteins contribute further to their stability in blood. This makes circulating miRNAs an attractive diagnostic tool. ${ }^{5}$

MiR-21 is one of the oncogenic microRNAs which promotes carcinogenesis via its anti apoptotic effect. MiRNA-21 gene is located at intron 10 on chromosome 17. Generally, miR-21 is observed to be up-regulated in cancers, and its suppression causes downsizing of tumor. It is also observed to contribute chemo resistance to the tumors. ${ }^{6}$ It also contributes in controlling genes like PTEN, TGF $\beta$, PDCD4 which are involved in the initial stage of cancers. ${ }^{7}$ Wei, 2011 and Yang Yu et al. experimentally validated PTEN as a target gene for miR-21. They found mir-21 inhibiting PTEN a tumor suppressor gene in prostate cancer cell line causing enhanced proliferation and invasion. ${ }^{8}$ It is proposed that miR21 is involved in carcinogenesis via its ability to inhibit apoptosis. ${ }^{9}$ To validate this and to find out the effect of miR-21 on its target genes Ma X et al. performed experiments on mice. They knocked out miR-21 allele in mouse ES. To achieve this they generated a vector which replaces precursor to miR-21 with neomycin (NEO)-resistance expression cassette. They observed miR-21 deficient mice to have significantly lower rate of papilloma development suggesting a protective effect against chemically induced skin carcinogenesis. ${ }^{10}$

Gombos $\mathrm{K}$ et al. also concluded from their work that mir-21 has a higher expression in Oral cancer. ${ }^{11}$ Expression of miR-21 in Oral cancer tumor tissue has been researched, however utility of the same as a plasma biomarker remains unknown. Objective of this study was to investigate expression of miR-21 in plasma of Oral cancer patients, to explore its diagnostic potential and to correlate the expression with various clinicopathological variables of Oral cancer.

\section{METHODS}

Present study was carried out at Ziauddin University and Karachi Institute of Radiotherapy and Nuclear Medicine (KIRAN). A total of 100 biopsy proven cases of Oral Squamous cell carcinoma were recruited via purposive sampling from Oncology department Ziauddin University between June 2013 and April 2017. Controls were carefully selected as healthy individuals of similar age group and gender as of tests. These controls were either healthy blood related family members of the cases or healthy individuals from same socio economic group. Board of Advanced studies and Ethical Review Committee of Ziauddin University gave approval of the protocol and written informed consent was obtained from all study subjects.

After conducting an interview and physical examination, $10 \mathrm{ml}$ of venous blood was collected in EDTA tubes. Samples were left for 15 mins and the supernatant buffy coat plasma was transferred to Eppendorf tube for RNA extraction.

RNA Extraction: RNA was extracted within 12$18 \mathrm{hrs}$ of blood collection and cDNA synthesized immediately from eluted RNA to avoid any chances of RNA degradation. RNA was extracted through Favorgen Nucleic acid extraction kit. Total RNA was extracted according to kit's protocol.

cDNA synthesis: cDNA was synthesized via Thermo fisher Revert Aid cDNA Synthesis kit. For cDNA preparation we added MMLV RT 4 $\mu \mathrm{l}$, dNTPs $2 \mu \mathrm{l}$, DTT $0.5 \mu \mathrm{l}$, Oligo dT primer $1 \mu \mathrm{l}$, reverted RT $1 \mu \mathrm{l}$, RNAse inhibitor $1 \mu \mathrm{l}$, distilled $\mathrm{H}_{2} \mathrm{O}$ $3.25 \mu \mathrm{l}$ and eluted miRNA $4 \mu \mathrm{l}$ to prepare a final volume of $17 \mu \mathrm{l}$. cDNA was prepared according to the set protocol as follows, incubation at $42^{\circ} \mathrm{C}$ for 50 $\min$, then $70^{\circ} \mathrm{C}$ for $10 \mathrm{~min}$ and then $4^{\circ} \mathrm{C}$. The cDNA was then stored at $-80^{\circ} \mathrm{C}$ until further analysis.

MiR-21 Expression: The same set of primers were used as previously reported by Wei et al. ${ }^{12}$ Following set of primers were used for sequencing 5'UAGCAGCACGUAAAUAUUGGCG3' for miR-16 5' UAGCUUAUCAGACUGAUGUUGA3' for miR21; and 5'UCACCGGGUGUAAAUCAGCUUG3' for cel-miR-39.

Considering low yield of miRNA in circulation its integrity and successful cDNA synthesis was evaluated by expression of GAPDH gene according to protocol by Thermo scientific. Samples which expressed a $496 \mathrm{~kb}$ band of GAPDH were further processed for miR-21 analysis. The amplification of internal control miR-16 was considered as a further proof of good quality miRNA extraction. For normalization of data mir-16 was used as endogenous controls. ${ }^{12}$

Real-time PCR was performed using SYBR green quantitative PCR reagent kit (Thermo scientific) on BioRad CFX96 analyzer. Each sample was analyzed in duplicate and appropriate negative controls were included. The $13 \mu \mathrm{l}$ PCR volume for amplification included $2 \mu \mathrm{l}$ of cDNA, $6.5 \mu \mathrm{l}$ of SYBR Green PCR Master Mix, $1.5 \mu l$ of primers and $3 \mu 1$ of $\mathrm{H}_{2} \mathrm{O}$. The 
reaction mixture was run at $95^{\circ} \mathrm{C}$ for $3 \mathrm{~min}$, followed by 40 cycles of $95^{\circ} \mathrm{C}$ for $5 \mathrm{~s}, 62^{\circ} \mathrm{C}$ for $35 \mathrm{~s}$ in which fluorescence was acquired. Rox was used as back ground noise on PCR program. Each sample was tested in duplicate. The expression levels of miR-21 were calculated utilizing the $2^{-\Delta \Delta} \mathrm{Ct}$ method. ${ }^{13}$

Statistical analysis: Statistical analysis of data was done via SPSS version 24. To express outcomes of qualitative variables frequency and percentages were used whereas mean with standard deviation was used for continuous variables. For comparison of variables chi square test was used for qualitative variables. Quantitative variables were compared using Students $t$ test in sets of two groups and one way ANOVA for more than two groups. To differentiate miR-21 between cases and controls Receiver Operating Curves (ROC) were generated. Pearson's correlation was used to correlate quantitative variables. A p-value of $<0.05$ was considered statistically significant.

\section{RESULTS}

A total of 100 samples (Oral cancer patients) and 100 controls were selected in the present study. Age and sex were not significantly different between cases and controls. There were 136 males and 64 females in both groups and average ct for both groups was same, $32.29 \pm 4.98$ for males vs. $31.77 \pm$ 5.4 for females, $\mathrm{p}=0.507$, CI $(-1.0164$ to -2.0510$)$. miR-21 expression was significantly higher among cases as compared to controls $29.4 \pm 5.3$ ct vs. $34.7 \pm$ $3.95 \mathrm{Ct}, \mathrm{p}<0.005$ (Fig.1). The cutoff for ct was set at
35 cycles, any expression below 35 ct was marked negative. To calculate relative expression $\Delta \mathrm{CT}$ or Livek method was used. $\Delta$ CT for cases was --5.39 whereas $\Delta \mathrm{CT}$ for controls was $-1.0371 . \Delta \Delta \mathrm{CT}$ of -6.4319 showed that cases had 6.3 times higher expression as compared to controls.

We did not observe any significant association between miR-21 expression and age, gender, ethnicity and smoking status. Out of 100 cases we had 67 Urdu speaking, 14 Sindi, 7 Baloch, 5 Punjabi, 3 Pashtoons and 4 others and expression of miR-21 was not different among various ethnic groups, $\mathrm{p}=0.869$.

Upon comparing miR-21 level with tumor characteristics we did not observe any significant association with Tumor grade, nodal status and tumor recurrence. However, among different tumor sizes, a significantly higher expression was observed if tumor size was $>4 \mathrm{~cm}, \mathrm{p}<0.001$. Metastatic tumors and locally invasive tumors also showed a significantly higher expression of miR-21 as shown in Table-I. Hence we suggest that circulating miR-21 originates from tumor mass as suggested by a positive association with tumor size, local invasion and metastasis.

We had 7 patients who presented with tumor recurrence and they had a similar expression to the patients who presented with primary tumor, $30.57 \pm$ 1.71 vs. $29.44 \pm 5.0, \mathrm{p}=0.557, \mathrm{CI}(-2.66115-4.90919)$.

To calculate sensitivity and specificity of the marker any expression seen after $35 \mathrm{Ct}$ was considered as negative. We found miR-21 had a sensitivity of $91 \%$ and specificity of $54 \%$ Receiver

Table-I: Relationship between Plasma miR 21 expression and Clinicopathological variables of Oral Cancer.

\begin{tabular}{|c|c|c|c|c|c|}
\hline Characteristic & & Number & $\begin{array}{c}\text { Mean } \pm \text { Standard } \\
\text { Deviation }\end{array}$ & Confidence Interval & $P$-value \\
\hline \multirow[t]{2}{*}{ Age } & $<40$ yrs & 36 & $29.91 \pm 4.2$ & -1.402 to 2.621 & 0.549 \\
\hline & $>40$ yrs & 64 & $29.30 \pm 5.18$ & & \\
\hline \multirow[t]{2}{*}{ Sex } & Male & 69 & $29.91 \pm 4.87$ & -0.840 to 3.313 & 0.240 \\
\hline & Females & 31 & $28.67 \pm 4.67$ & & \\
\hline \multirow[t]{2}{*}{ Smoking status } & Yes & 66 & $29.98 \pm 4.7$ & -0.696 to 3.35 & 0.196 \\
\hline & No & 34 & $28.65 \pm 4.94$ & & \\
\hline \multirow[t]{3}{*}{ Grade } & Well differentiated & 14 & $29.5 \pm 4.8$ & & 0.490 \\
\hline & Moderate differentiated & 70 & $29.8 \pm 4.6$ & & \\
\hline & Poor differentiated & 16 & $28.2 \pm 5.6$ & & \\
\hline \multirow[t]{2}{*}{ Tumor Size } & $<4 \mathrm{~cm}$ & 50 & $31.37 \pm 3.69$ & $1.91-5.48$ & $<0.001$ \\
\hline & $\geq 4 \mathrm{~cm}$ & 50 & $27.67 \pm 5.1$ & & \\
\hline \multirow[t]{2}{*}{ Nodal Status } & Node negative & 6 & $29.18 \pm 4.67$ & -4.43 to 3.70 & 0.859 \\
\hline & Node Positive & 94 & $29.5 \pm 4.8$ & & \\
\hline \multirow[t]{2}{*}{ Metastasis } & No & 93 & $29.8 \pm 4.7$ & 1.53 to 9.41 & 0.007 \\
\hline & Yes & 6 & $24.4 \pm 4.42$ & & \\
\hline \multirow[t]{2}{*}{ Local Invasion } & No & 81 & $30.06 \pm 4.54$ & 0.4124 to 5.214 & 0.022 \\
\hline & Yes & 19 & $27.2 \pm 5.5$ & & \\
\hline
\end{tabular}




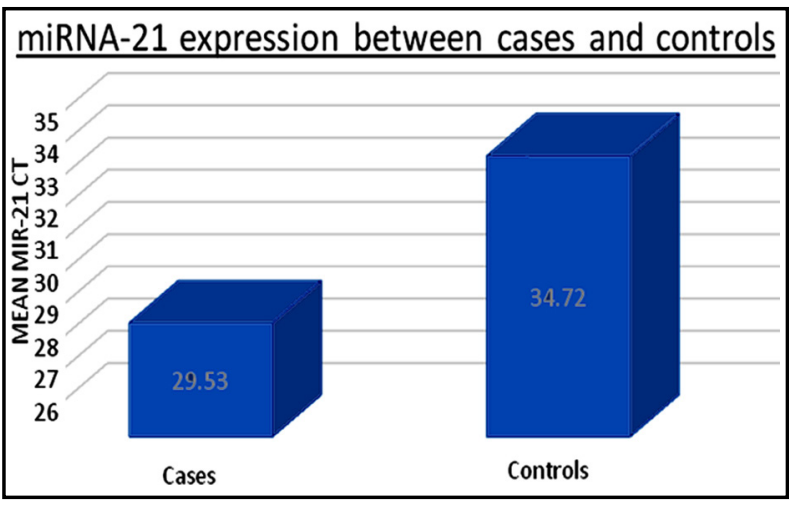

Fig 1:MiR- 21 is upregulated in Oral cancer as indicated by lower CT. MiR 21 expression in serum of Oral cancers and healthy controls determined by qRT-PCR (quantitative reverse transcription polymerase chain reaction. ${ }^{*} \mathrm{p} \leq 0.05$.

operating characteristic (ROC) curve was generated based on ct values of miR-21. The Area under curve (AUC) was $0.829, \mathrm{p}<0.001$ suggesting that miR-21 can be used as a marker to discriminate Oral cancer from normal healthy control (Fig.2).

\section{DISCUSSION}

A higher expression of miR-21 in patients with oral squamous cell carcinoma was observed. miR-21 is amongst the first miRNA observed to be expressed in serum. Arantes et al. investigated role of miRNAs in Head and neck cancer by extracting miRNA from tumor tissue. To start with, they screened 15 cases on a microarray to identify miRNAs deregulated in Oral cancer and found mir-21 to be over expressed. They validated their findings on a larger set of patients using miR-21 specific primers on real time PCR and found miR-21 to be an independent prognostic factor in a model adjusted for age, tumor site and tumor resectability. ${ }^{14}$ Similarly, Hedback $\mathrm{N}$ et al. investigated the expression of miR 21 in paraffin embedded tumor tissue and adjacent normal tissue from oral cancer cases using in situ hybridization. They observed a higher expression not only in cancer cells but also in tumor stroma and tumor blood vessels. Higher expression in tumor stroma was found to have a negative prognostic value on a multivariate analysis after adjusting for $\mathrm{N}$ stage and perineural invasion. ${ }^{15}$

Another study by Gombos et al. also checked for the miR-21 expression in tumor tissue and compared expression in the adjacent normal tissue. Upon comparing the expression through paired test they observed a significant over expression of miR21 and receiver operating characteristic (ROC) curve showed a sensitivity and specificity of above $90 \%{ }^{11}$

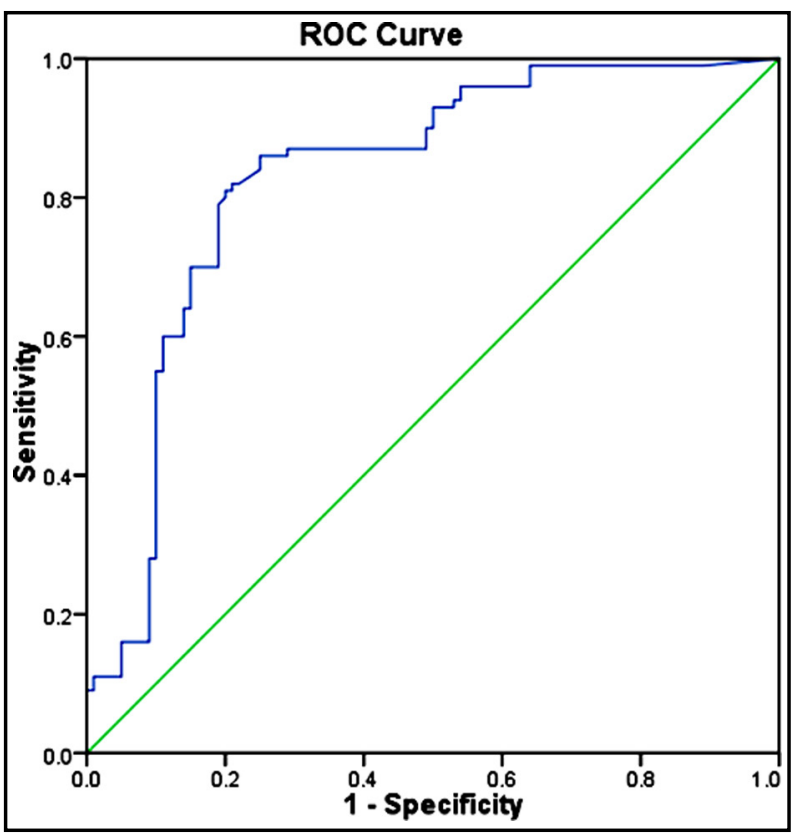

Fig.2: Receiver operating characteristic (ROC) curve analysis for miR-21 based on RT-qPCR data in discriminating oral cancer from controls. Area under curve $0.829, \mathrm{p}<0.001$.

Major research done in the field of miRNA in Oral cancers is either on the cell lines or surgically resected tumor specimens. One major disadvantage of a tissue based biomarker is invasiveness associated with the procedure. To overcome this identification of circulating biomarkers has gained considerable momentum. However, assuming the low concentration of miRNA in circulation very little has been done to search for circulating miRNAs in blood. Tachiban et al. used a high throughput highly sensitive array to determine circulating miRNA profile. On a profile of 1211 human miRNAs 20 showed more than two fold change compared to healthy controls. Of these 20, 16 were up regulated and 4 down regulated. Mir223 showed the most significant up regulation so it was further validated on real time PCR and was proposed as a potential biomarker for diagnosis of oral cancer. ${ }^{16}$ Tachibana et al and Reis et al discovered miR-186, miR-3651 and miR- 494 to be deregulated in serum of OSCCA. . $^{16,17}$

We also report a higher expression of miR21 but, interestingly we measured miR-21 in serum of OSCCA patients making it possible to chalk out patient's prognosis via a less invasive serum biomarker. Shah $\mathrm{S}$ et al. conducted a comprehensive review of researches published on miR-21 in Oral squamous cell carcinoma and concluded a negative correlation with prognosis. 
In addition, they highlighted the therapeutic potential of inserting anticancer construct in miR21 promotor region. ${ }^{18}$

Unlike breast, prostate, ovarian and liver cancer research has failed to discover a biomarker which could help in diagnosis and predict recurrence in oral cancer. The need is to dig deep in to the molecular events involved in oral cancer and to search for molecular biomarkers which could enable timely diagnosis and predict recurrence and metastasis.

Limitations of this study: It is selection from a single center which is likely to contribute to selection bias and hence compromise on generalizability of results. Secondly we only compared expression of miR-21 between cases and controls, adding another group of patients with pre malignant Oral lesions would have served to add important information. Future multicenter studies with a larger sample size and premalignant patients would help in exploring its role as potential screening marker in oral cancer.

\section{CONCLUSION}

Our findings suggest that serum miR-21 levels reflect tissue mir21 expression and hence it may be used as a non invasive tool for predicting tumor invasion and metastasis.

\section{Grant Support \& Financial Disclosures: None.}

Conflict of interest: None.

\section{REFERENCES}

1. Sarwar MR, Saqib A, Schumacher U. Cancer prevalence, incidence and mortality rates in Pakistan in 2012. Cogent Med. 2017;4:1. doi: 10.1080/2331205X.2017.1288773.

2. Locally advanced squamous cell carcinoma of the head and neck. Head and neck cancer union for international cancer control. Review of cancer Medicines on the WHO list of Essential Medicines. 2014.

3. Corsini LR, Bronte G, Terrasi M, Amodeo V, Fanale D, Fiorentino $\mathrm{E}$, et al. The role of microRNAs in cancer: diagnostic and prognostic biomarkers and targets of therapies. Expert Opin Ther Targets. 2012;16(Suppl 2):S103-S109. doi: 10.1517/14728222.2011.650632

4. Allegra A, Alonci A, Campo S, Penna G, Petrungaro A, Gerace D, et al. Circulating microRNAs: new biomarkers in diagnosis, prognosis and treatment of cancer. Int J Oncol. 2012;41(6):1897-1912. doi: 10.3892/ijo.2012.1647

5. Arroyo JD, Chevillet JR, Kroh EM, Ruf IK, Pritchard CC, Gibson $\mathrm{DF}$, et al. Argonaute2 complexes carry a population of circulating microRNAs independent of vesicles in human plasma. Proc Natl Acad Sci. 2011;108(12):5003-5008. doi: 10.1073/pnas.1019055108

6. Selcuklu SD, Yakicier MC, Erson AE. MIR21 (microRNA 21). Atlas Genet Cytogenet Oncol Haematol. 2007;3:440-447.

7. Xu LF, Wu ZP, Chen Y, Zhu QS, Hamidi S, Navab R. MicroRNA-21 (miR-21) regulates cellular proliferation, invasion, migration, and apoptosis by targeting PTEN, RECK and Bcl-2 in lung squamous carcinoma, Gejiu City, China. PloS One. 2014;9(8):e103698. e103698. doi: 10.1371/journal.pone.0103698

8. Yang Y, Guo JX, Shao ZQ. miR-21 targets and inhibits tumor suppressor gene PTEN to promote prostate cancer cell proliferation and invasion: An experimental study. Asian Pac J Trop Med 2017;10(1):87-91. doi: 10.1016/j.apjtm.2016.09.011
9. Li C, Hu Y, Deng X, Shen X, Mao N, Xiong T, et al. Anti-apoptotic roles of MicroRNA-21 via activating PTEN/PI3K/Akt pathway in lipopolysaccharide-induced acute lung injury. Int J Clin Exp Pathol. 2017;10(4):4339-4347.

10. Ma X, Kumar M, Choudhury SN, Buscaglia LE, Barker JR, Kanakamedala $\mathrm{K}$, et al. Loss of the miR-21 allele elevates the expression of its target genes and reduces tumorigenesis. Proc Nat Acad Sci. 2011;108(25):10144-10149. doi:10.1073/pnas.1103735108/-/ DCSupplementa

11. Gombos K, Horvath R, Szele E, Juhasz K, GoCZE KA, Somlai K, et al. miRNA expression profiles of oral squamous cell carcinomas. Anticancer Res. 2013;33(4):1511-1517.

12. Wei J, Liu LK, Gao W, Zhu CJ, Liu YQ, Cheng T, et al. Reduction of plasma microRNA-21 is associated with chemotherapeutic response in patients with non-small cell lung cancer. Chin J Cancer Res. 2011;23(2):123-128. doi: 10.1007/s11670-011-0123-2

13. Schmittgen TD, Livak KJ. Analyzing real-time PCR data by the comparative C(T) method. Nat Protoc. 2008;3(6):1101-1108. doi: 10.1038 /nprot.2008.73

14. Arantes LM, Laus AC, Melendez ME, de Carvalho AC, Sorroche $\mathrm{BP}$, De Marchi PR, et al. MiR-21 as prognostic biomarker in head and neck squamous cell carcinoma patients undergoing an organ preservation protocol. Oncotarget. 2017;8(6):9911. doi: 10.18632/ oncotarget.14253

15. Hedback N, Jensen DH, Specht L, Fiehn AM, Therkildsen MH Friis-Hansen L, et al. MiR-21 expression in the tumor stroma of oral squamous cell carcinoma: an independent biomarker of disease free survival. PloS One. 2014;9(4):e95193. doi: 10.1371/journal. pone. 0095193

16. Tachibana H, Sho R, Takeda $Y$, Zhang $X$, Yoshida $Y$, Narimatsu $\mathrm{H}$, et al. Circulating miR-223 in oral cancer: its potential as a novel diagnostic biomarker and therapeutic target. PloS one. 2016;11(7):e0159693. doi: 10.1371/journal.pone.0159693

17. Ries J, Vairaktaris E, Agaimy A, Kintopp R, Baran C, Neukam FW, et al. miR-186, miR-3651 and miR-494: potential biomarkers for oral squamous cell carcinoma extracted from whole blood. Oncol Rep. 2014;31(3):1429-1436. doi: 10.3892/or.2014.2983

18. Shah S, Jadhav K, Shah V, Gupta N, Dagrus K. miRNA 21: Diagnostic Prognostic and Therapeutic Marker for Oral Cancer. MicroRNA. 2016;5(3):175-179. doi: 10.2174/2211536605666160919115323

\section{Author`s Contribution:}

NM and MH did collection, designed, manuscript writing, and editing of manuscript.

MS, SM and AK did data collection, sample run, analysis, and editing of manuscript.

QJ and AH did review, editing and final approval of manuscript.

Authors:

1. Dr. Nosheen Mahmood, MBBS, M.Phil, M.Phil Pathology, PhD Fellow.

Department of Pathology,

2. Dr. Muhammad Hanif, PhD (Microbiology) Head of Department (Pr. Scientist) Clinical labs \&

Molecular Biology Lab,

3. Dr. Akhtar Ahmed, FCPS

4. Dr. Qamar Jamal, PhD Pathology,

Professor of Pathology,

5. Dr. Shamim Mushtaq, PhD.

Associate Professor,

6. Dr. Adnan Khan, PhD.

7. Mr. Muhammad Saqib, M.Sc.

1,4,5: Ziauddin Medical University, Karachi, Pakistan

2,3,6,7: Karachi Institute of Radiotherapy and Nuclear Medicine (KIRAN), Karachi, Pakistan. 